\title{
BMJ Open Impact of a patient-specific national programme aimed at increasing the use of emollient moisturisers to reduce the risk of skin tears: a longitudinal cohort study
}

\author{
Anna K Moffat (1D , ${ }^{1}$ Kerrie P Westaway, ${ }^{1}$ Jemisha Apajee, ${ }^{1}$ Oliver Frank, ${ }^{2}$ \\ Russell Shute, ${ }^{3}$ Clare Weston, ${ }^{4}$ Natalie Blacker, ${ }^{1}$ Vanessa T Le Blanc, ${ }^{1}$ \\ Lisa M Kalisch Ellett, ${ }^{1}$ Nicole L Pratt, ${ }^{1}$ Elizabeth Ellen Roughead ${ }^{1}$
}

To cite: Moffat AK, Westaway KP, Apajee J, et al. Impact of a patient-specific national programme aimed at increasing the use of emollient moisturisers to reduce the risk of skin tears: a longitudinal cohort study. BMJ Open 2020;10:e039579. doi:10.1136/ bmjopen-2020-039579

- Prepublication history for this paper is available online. To view these files, please visit the journal online (http://dx.doi. org/10.1136/bmjopen-2020039579).

Received 21 April 2020 Revised 15 September 2020 Accepted 09 0ctober 2020
Check for updates

(C) Author(s) (or their employer(s)) 2020. Re-use permitted under CC BY-NC. No commercial re-use. See rights and permissions. Published by BMJ.

For numbered affiliations see end of article.

Correspondence to

Dr Anna K Moffat;

annakmoffat@gmail.com

\section{ABSTRACT}

Objectives To evaluate the impact of a patient-specific national programme targeting older Australians and health professionals that aimed to increase use of emollient moisturisers to reduce to the risk of skin tears.

Design A prospective cohort intervention.

Participants The intervention targeted 52778 Australian Government's Department of Veterans' Affairs patients aged over 64 years who had risk factors for wound development, and their general practitioners (GPS) $(n=14178)$.

Outcome measures An interrupted time series model compared the rate of dispensing of emollients in the targeted cohort before and up to 23 months after the intervention. Commitment questions were included in self-report forms. Results In the first month after the intervention, the rate of claims increased 6.3-fold (95\% Cl: 5.2 to $7.6, p<0.001$ ) to 10 emollient dispensings per 1000 patients in the first month after the intervention. Overall, the intervention resulted in 10905 additional patient-months of treatment. The increased rate of dispensing among patients who committed to talking to their GP about using an emollient was six times higher (rate ratio: $6.2,95 \% \mathrm{Cl}$ : 4.4 to 8.7 ) than comparison groups.

Conclusions The intervention had a sustained effect over 23 months. Veterans who responded positively to commitment questions had higher uptake of emollients than those who did not.

\section{INTRODUCTION}

Skin tears are traumatic wounds caused by shear, friction and/or blunt force resulting in separation of the skin layers. ${ }^{1}$ They cause considerable discomfort and pain, have the potential to cause infection and become chronic wounds, and reduce quality of life in a vulnerable population. ${ }^{2}$

A randomised controlled trial conducted during 2011-2012 in 14 aged care facilities in Western Australia investigated the effectiveness of applying skin emollient moisturiser two times per day to residents' arms and legs to reduce skin tear incidence over a 6 -month period. ${ }^{3}$ The
Strengths and limitations of this study

A strength of this study is that it describes a nationwide intervention providing targeted patient-specific information to patients' primary general practitioner.

- A strength of this study is the involvement of patient and healthcare providers and ability to track dispensing of prescribed emollient moisturisers on a national level.

- This study is limited due to the inability to identify skin tears in the data to determine the impact of the intervention on health outcomes.

emollient moisturiser used was a commercially available, $\mathrm{pH}$ neutral, perfume-free lotion. The Skin Tear Audit Research Classification System was used to classify skin tears according to their characteristics and severity. ${ }^{3}$ The average monthly incident rate of skin tears in the intervention group that used an emollient moisturiser was almost half that of the comparison group (5.76 skin tears per 1000 occupied bed days in the intervention group compared with 10.57 per 1000 occupied bed days in the control group). ${ }^{3}$

Building on these results, a prospective intervention study conducted between January 2013 and December 2013 in a large Queensland private hospital evaluated the effectiveness of applying emollient moisturiser two times per day to reduce the incidence of skin tears in older inpatients. ${ }^{4}$ Seven hundred and sixty-two patients aged 73-100 years from two wards with a history of a high incidence of reported skin tears received two times per day application of a commercially available, non-perfumed, $\mathrm{pH}$-friendly emollient moisturiser to their arms and legs. ${ }^{4}$ Incidence of skin tears was compared with a historical sample of 415 patients aged 
69-96 years from the same two wards. Of the 762 patients in the intervention group, $60(8 \%)$ developed at least one skin tear, while $44(11 \%)$ of the 415 patients in the historical group had at least one skin tear, equivalent to 2.26 fewer skin tears per 1000 occupied bed days in the intervention group compared with controls. ${ }^{4}$ Patients in the intervention group were older, had greater cognitive impairment and a greater degree of incontinence than patients in the usual care control group, all of which might have been expected to make skin tears more likely. ${ }^{4}$ Both studies assessing the effectiveness of the application of an appropriate emollient moisturiser to reduce skin tears resulted in positive outcomes, and highlight the benefits associated with a relatively low-cost, simple intervention to reduce the incidence of skin tears in older people. ${ }^{34}$

Quality improvement interventions to increase the use of emollient moisturisers in older people are likely to significantly reduce the incidence of skin tears; however, achieving the desired behavioural outcomes in quality improvement interventions can be challenging. ${ }^{56}$

Interventions that ask participants about their commitment to perform certain actions in relation to a behaviour can influence the uptake of that behaviour. ${ }^{7}$ Research shows commitment questions can increase target behaviours in accordance with the principles of consistency, where people aim to maintain a certain approach across their words, beliefs, attitudes and actions. ${ }^{78} \mathrm{~A}$ positive response to commitment questions has shown to increase uptake of targeted health services in national, multifaceted interventions. ${ }^{8}$ For example, the monthly rate of Home Medicines Reviews (HMRs) was increased among responders who answered positively to a commitment question to talk with their general practitioner (GP) about having an HMR (rate ratio (RR): 2.64, 95\% CI: 2.39 to 2.92), in comparison to non-responders or those who answered negatively. Similar results have been seen following interventions aiming to increase the uptake of dose administration aid use (RR: 2.53, 95\% CI: 2.29 to 2.79), General Practice Management Plans (RR: 1.30, 95\% CI: 1.14 to 1.48 ) and care plans among patients with diabetes (RR: $1.47,95 \% \mathrm{CI}$ : 1.24 to 1.75$)^{8}$.

In 2017, we implemented a quality improvement intervention which aimed to increase the use of emollient moisturisers as a strategy to reduce to the risk of skin tears in the older Australian veteran community. The aim of this study is to evaluate the impact of that national health promotion programme on the rate of dispensing of emollient moisturisers in the 23 months following the intervention.

\section{METHODS}

The Veterans' Medicines Advice and Therapeutics Education Services (Veterans' MATES) programme, funded by the Australian Government's Department of Veterans' Affairs (DVA), is a national, data-driven, patient-specific, health promotion programme that aims to improve the use of medicines and related health outcomes in the Australian veteran community. ${ }^{9}$ The programme accesses the DVA health claims database to identify health-related or medicine-related issues in the veteran community. The database contains details of all prescription medicines, medical, dental and allied health services and hospitalisations provided to veterans and their eligible dependents as well as gender, date of birth, date of death and family status. Of the estimated 196000 veterans alive in $2017,154600(79 \%)$ were aged 60 years and over. ${ }^{10}$ There were an estimated 82600 veterans aged 80 years and over in $2017 .^{10}$

The Veterans' MATES programme is underpinned by the behavioural and health promotion theories, Social Cognitive Theory, ${ }^{11}$ the Transtheoretical Model, ${ }^{12}$ the PrecedeProceed Model ${ }^{13}$ and incorporates the use of commitment questions as outlined in persuasive communication ${ }^{7}$ and the Nudge Theory ${ }^{14}$ to encourage veterans participating in the programme to acquire and maintain new behavioural patterns. ${ }^{9}$ A key feature of the programme is individual patient-specific feedback provided to GPs about potential medicine-related or health-related problems among their DVA patients (see figure 1); this is accompanied by advice for GPs and a request to review the patient. Supportive evidencebased educational materials are provided, and health professionals are supported by a national call centre staffed by pharmacists. Other health professional groups, including pharmacists and staff at aged-care facilities are also mailed the educational materials to re-enforce messages among the healthcare team.

DVA patients receive educational materials specifically tailored to them based on their recorded history and they also have access to a clinical support phone line staffed by pharmacists at a national call centre. Stakeholder self-report forms assess participation satisfaction, changes in awareness and knowledge, and self-reported behavioural intentions.

\section{Skin tears intervention}

This intervention targeted 52778 DVA patients aged 65 years or older who were living in the community or in an aged care facility and had risk factors for wound development (see figure 1), 14178 GPs who were identified as the DVA patient's primary GP, 8363 pharmacists and 2504 directors of aged care facilities in June 2017. Messages in the educational materials to healthcare professionals emphasised the effectiveness of the application of an appropriate emollient moisturiser two times per day to reduce the risk of skin tears. Messages in the educational materials to their DVA patients provided practical tips on how to look after their skin, including the application of an appropriate emollient moisturiser two times per day to reduce the risk of skin tears. The brochure for patients advised that they may be eligible to receive an emollient moisturiser subsidised by Australia's national Repatriation Pharmaceutical Benefits Scheme (RPBS). ${ }^{15}$

Patients were asked to complete a one-page response form that included a question regarding whether they were likely to talk to their GP about which emollient moisturiser was appropriate for their use, we asked: effective moisturisers are subsidised through the RPBS). Do you intend to talk to your doctor about which moisturiser to use? Respondents could select from the answers: Yes, No, Unsure or I already use a 


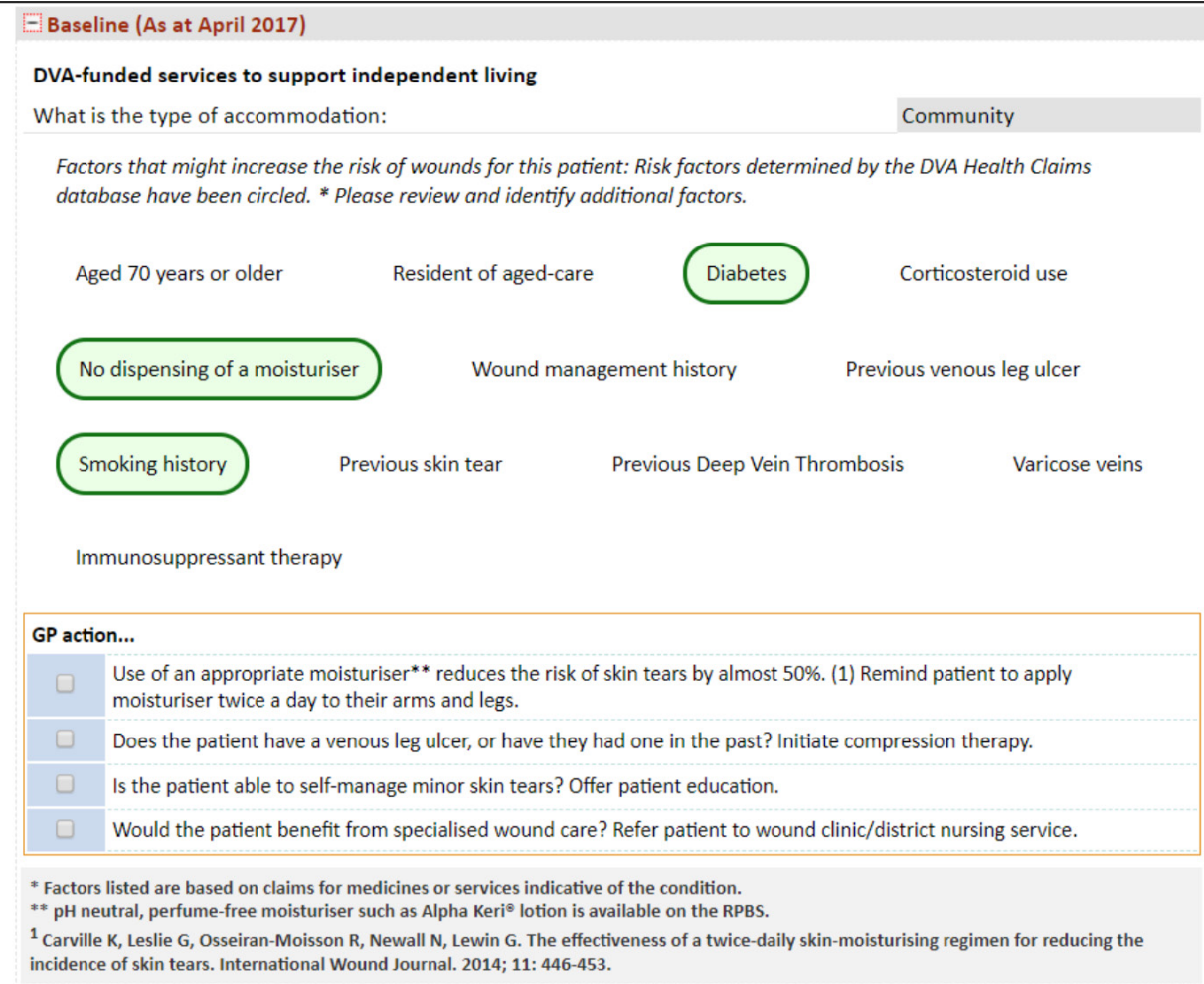

Figure 1 Patient-specific feedback provided to each GP with information about their patient and advice to consider if appropriate. DVA, Department of Veterans' Affairs; GP, general practitioner.

moisturiser. Data from the patients' returned response forms were scanned into comma separated files by Australia Post.

To evaluate the impact of the intervention on dispensing of emollient moisturisers, we used DVA health claims data. Emollient moisturisers are coded in the DVA dataset according to the classification and the Schedule of Pharmaceutical Benefits item codes. ${ }^{16}$ We assessed monthly rate of claims for emollient moisturisers among all DVA patients aged 65 years and older.

The impact of the intervention was measured by comparing the rate of dispensing of emollient moisturisers in the population targeted in the intervention, before and after the intervention. The monthly rate of dispensing of emollient moisturisers was calculated as the number of unique DVA patients dispensed at least one emollient moisturiser in the month divided by the number of DVA patients targeted who were still alive in that month. The rate is expressed as the rate per 1000 DVA patients per month.

To determine whether the rate of dispensing of emollient changed after the intervention, we used proc autoreg in SAS V.9.4 to fit an interrupted time series model with autoregressive errors ${ }^{17}$ and adjusted for seasonality in the data. The intervention point was July 2017. We applied a log transformation to the rate of dispensing to ensure that the predicted values were bounded below by zero. We estimated the preintervention trend (July 2014-June 2017), the change in level at the time of the intervention (July 2017) and the change in trend post-intervention (July 2017-May 2019) compared with pre-intervention. We included indicator variables for the months of January-June and August-December in the model to adjust for seasonality. July was the reference month. We also included another indicator variable in the model to estimate the observed peak in the data in August 2017, 1 month after the intervention. The autoregressive terms were selected automatically using the backstep option in proc autoreg. The number of additional patient dispensings in the 23 months following the intervention was calculated as the difference between the number of patients dispensed an emollient moisturiser in the time period July 2017-May 2019 and the predicted number of patients who would have been dispensed an emollient moisturiser in the same time period if the intervention had not occurred. To calculate the additional patient-months of treatment, the total number of additional patient dispensings was multiplied by 3 . We assumed that one dispensing would provide 3 months of treatment as $75 \%$ of patients return for a refill within 3 months of dispensing. ${ }^{18}$

To determine the impact of the commitment questions on the dispensing of emollient moisturisers, we stratified the time series by the patients' response to the question: do you intend to talk to your doctor about which moisturiser to use?, as reported in the self-response form. The average rates of claims post-intervention were compared across the response groups using a log-binomial model using those who did not respond as the reference group. Proc genmod in SAS V.9.4 was used.

\section{Patient or public involvement}

The Veterans' MATES programme involves a number of stakeholder groups in the design and implementation of the interventions. These stakeholder groups include veterans 


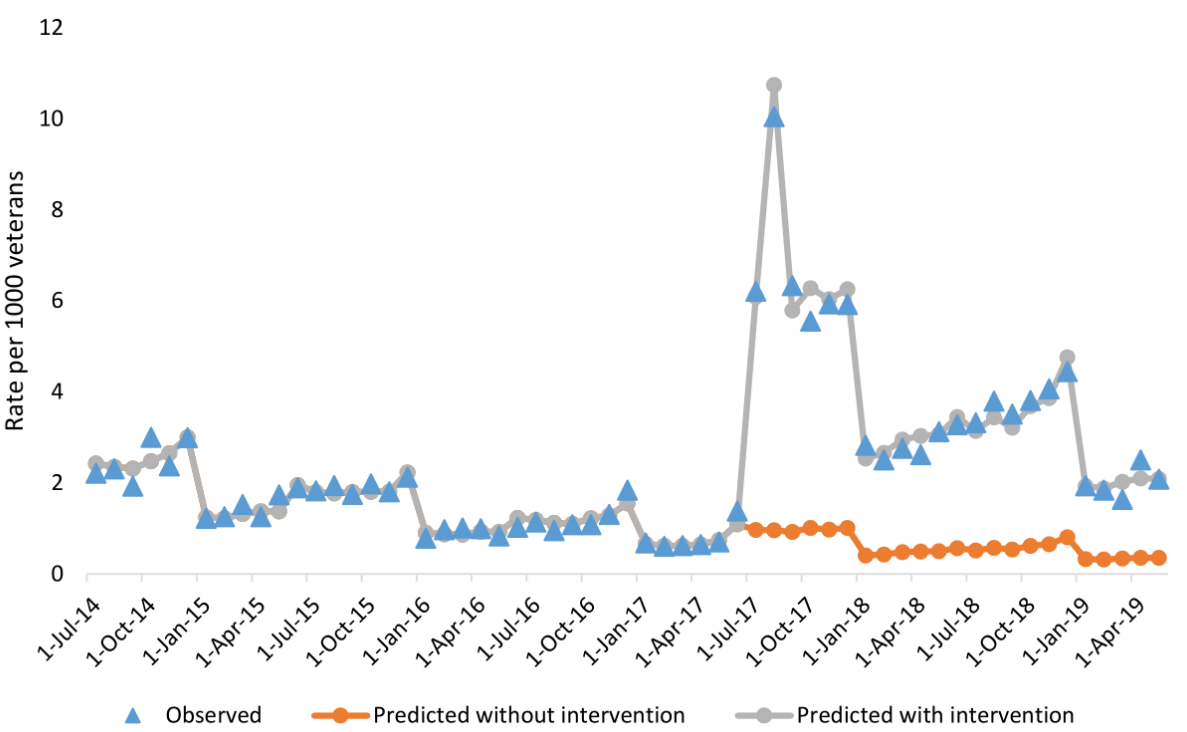

Figure 2 Observed and modelled rate of patients dispensed emollient moisturisers in the targeted DVA patients.

who access services and subsidies through DVA, as well as expert and medical stakeholder groups.

\section{RESULTS}

This intervention targeted 52778 DVA patients. Figure 2 shows the rate of dispensing of emollient moisturisers in the targeted DVA patients along with the predicted rate of claims with and without the intervention. Table 1 shows that prior to the intervention the rate of dispensing declined by $2.8 \%$ per month (95\% CI: $-3.2 \%$ to $-2.4 \%$ ), starting from 2.2 dispensings per 1000 DVA patients in July 2014 and declining to 0.7 per 1000 in May 2017 (figure 2). In the month of the intervention (July 2017), the rate of claims increased 6.3-fold followed by a further 1.8-fold increase in August 2017. Six months after the intervention, the rate remained high at six dispensings per 1000 patients. After 18 months post-intervention, the increased rate of dispensing of emollient moisturisers was sustained at 4.5 dispensings per 1000 patients compared with the predicted rate of 1 dispensing per 1000 patients per month. Based on this model, we estimate that the Veterans' MATES intervention resulted in an additional 3635 dispensings and an additional 10905 patientmonths of treatment with an emollient moisturiser in the 2 years following the intervention.

The gradual increase in the observed and fitted rates over the course of the calendar year is likely due to the PBS safety net implemented by the Australian Government to ensure affordability of medicines to families in a calendar year. Patients receive subsidised medicines (including emollients under the RPBS) free of charge once the safety net is reached. It is common for rates to increase over the course of the calendar year as patients obtain extra quantities of medicines to stockpile for the new year when standard prices resume.

Figure 3 shows the rate of dispensing of emollient moisturisers stratified by the response of yes, no/unsure or no response in relation to whether DVA patients intended to talk with their GP about an appropriate emollient moisturiser to use. There were 8162 DVA patients who responded that they would talk to their GP, 3977 who indicated that they would not talk to their GP or that they were unsure and 40639 who did not respond. The increase in the rate of dispensings

Table 1 Results of segmented regression model assessing the impact of the Veterans' MATES intervention on emollient dispensing

\begin{tabular}{|c|c|c|c|}
\hline & $\begin{array}{l}\text { Exponential parameter } \\
\text { estimate (trend as \% } \\
\text { change per month) }\end{array}$ & $95 \% \mathrm{Cl}$ & $P$ value \\
\hline Intercept & 2.50 & 2.27 to 2.78 & $<0.0001$ \\
\hline Pre-intervention trend & $0.97(-2.8 \%)$ & 0.97 to $0.98(-3.2 \%$ to $-2.4 \%)$ & $<0.0001$ \\
\hline Change in level in the month of the intervention & 6.29 & 5.19 to 7.61 & $<0.0001$ \\
\hline $\begin{array}{l}\text { Additional change in the level in for the month of } \\
\text { August } 2017 \text { only }\end{array}$ & 1.78 & 1.32 to 2.39 & 0.0003 \\
\hline Change in trend after the Veterans' MATES intervention & $1.00(-0.4 \%)$ & 0.99 to 1.01 & 0.43 \\
\hline
\end{tabular}

MATES, Medicines Advice and Therapeutics Education Services. 


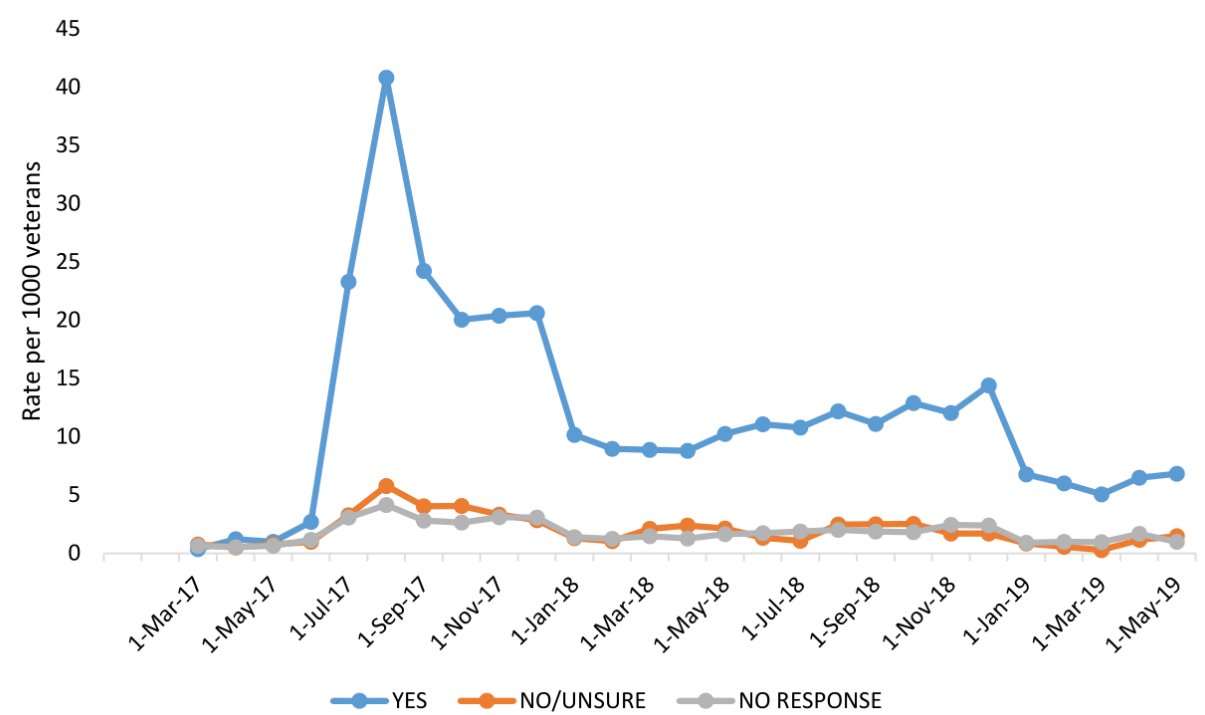

Figure 3 Rate of patients dispensed an emollient moisturiser in the targeted DVA patients grouped by reported intention to talk to their GP about an appropriate emollient moisturiser to use.

post-intervention was highest among those who indicated they would talk to their GP.

Compared with the rate of dispensing for DVA patients who indicated that they would not talk to their GP or were unsure, the rate of dispensing in those who indicated they would talk to their GP was more than six times greater (rate ratio $=6.2,95 \%$ CI 4.4 to 8.7 ) than those who did not respond, and a sustained increase was observed over the whole follow-up. Those who did not respond were similar to those who responded that they would not talk to their GP or were unsure ( $\mathrm{RR}=1.09,95 \% \mathrm{CI}: 0.8$ to 1.5 ).

\section{DISCUSSION}

Our nation-wide intervention was successful in increasing the use of emollient moisturisers in older Australians, resulting in an additional 10905 patient-months of treatment. In keeping with behaviour change theory regarding patient engagement and use of commitment questions, the largest increase to emollient moisturiser use was seen in people who returned a response form for the intervention which indicated they intended to speak to their GP about using an emollient moisturiser. The intervention was informed by two studies, which showed the benefits associated with a relatively low-cost, simple intervention of applying an emollient moisturiser two times per day to reduce the incidence of skin tears in older people in a hospital and aged care setting. ${ }^{34}$ Although we are unable to assess whether our intervention resulted in reduced skin tears for participants, we were able to show the rate of dispensing of emollient moisturisers increased substantially after implementing the intervention, and that there was a sustained increased rate of dispensing 18 months after the intervention. Strategies used in the DVA patients' educational brochure and response form are likely to have facilitated the positive behaviour change. Behaviour theory suggests that asking questions about target behaviours makes it more likely that people will perform the targeted behaviour, and that people who make an initial commitment which is active, public or freely chosen, are more likely to behave consistently with their initial commitment. ${ }^{7}$ 'Call to action' questions, a form of commitment question, were used in our study as an active strategy to influence behaviour change. Having a specific and practical message in the DVA patients' educational brochure about how to reduce the risk of skin tears, and 'call to action' or 'commitment' questions in the response form, were likely to have prompted patients to be consistent and say 'yes' to both questions: 'since reading the brochure, are you more likely to moisturise twice a day?' and 'do you intend to talk with your doctor about which moisturiser to use?' A sixfold increase in the rate of dispensing of emollient moisturisers in the 2 months after the intervention in patients who said they intended to speak with their GP about an appropriate emollient moisturiser compared with those who did not report an intention to speak with their GP indicates the call to action questions helped facilitate the behaviour change. The simplicity associated with commitment questions ${ }^{78}$ suggest asking straightforward questions about behaviour and intentions are a beneficial addition to the targeted educational intervention.

The strength of our study lies in its national involvement of the older community and healthcare professionals, and the ability to track dispensing of prescribed emollient moisturisers on a national level. Our results are likely to be generalisable to older Australians living in the community and in aged care facilities. There are limitations to this study. The patients who responded positively may have been more likely to have engaged in the behavioural outcome regardless of the commitment questions; however, given, respondents who indicated no or unsure had some level of engagement with the programme and results for this strata were no different to non-responders, responding as a factor did not appear to be 
influential. A further limitation of the study was the inability to identify skin tears in the data to determine the impact of the intervention on health outcomes. However, prior research has shown that the use of an emollient moisturiser reduces the incidence of skin tears in older people, ${ }^{34}$ so it is likely that the increased use of moisturisers as a result of our intervention was associated with improved health outcomes. While the administrative claims data used in this study has advantages, including large sample size, it is also unable to capture behavioural data, including whether and how the emollient was used by the patients who obtained it following the intervention. Furthermore, it is possible that patients accessed privately funded emollients which are not captured in our data, despite being eligible for subsidised items. Future work should explore the impact of a targeted intervention on increasing emollient use where skin tear outcome data are available.

Our study demonstrates that a targeted patient-specific programme is useful for increasing the use of emollient moisturisers in older people. Commitment questions are an important driver of health behaviour change.

\section{Author affiliations}

'Department of Clinical and Health Sciences, Quality Use of Medicines and Pharmacy Research Centre, University of South Australia, Adelaide, South Australia, Australia

${ }^{2}$ Discipline of General Practice, Adelaide Medical School, The University of Adelaide, Adelaide, South Australia, Australia

${ }^{3}$ Veterans' MATES Reference Group, University of South Australia, Adelaide, South Australia, Australia

${ }^{4}$ Veterans' MATES Reference Group, NPS MedicineWise, Strawberry Hills, South Australia, Australia

Acknowledgements This article is a part of an evaluation conducted by the Australian Government's Department of Veterans' Affairs (DVA), Veterans' Medicines Advice and Therapeutics Education Services (Veterans' MATES) Programme. The Australian Government's DVA Veterans' MATES Programme is provided by the Quality Use of Medicines and Pharmacy Research Centre, Sansom Institute, University of South Australia, in association with: Discipline of General Practice, University of Adelaide; Discipline of Public Health, University of Adelaide; NPS MedicineWise; Australian Medicines Handbook; and Drug and Therapeutics Information Service. Veterans' MATES Programme materials are available at: www. veteransmates.net.au.

Contributors AKM and JA: study design, drafting the manuscript and data analysis. KPW: study design and drafting the manuscript. OF, RS, CW, NB, VTLB and LMKE: study design and critical revision of the manuscript for important intellectual content. NLP and EER: study design, critical revision of the manuscript for important intellectual content and data analysis. All authors read and approved the final manuscript.

Funding This research was funded by the Australian Government's Department of Veterans' Affairs as part of the delivery of the Veterans' Medicines Advice and Therapeutics Education Services project.

Competing interests None declared.

Patient and public involvement Patients and/or the public were involved in the design, or conduct, or reporting, or dissemination plans of this research. Refer to the Methods section for further details.

Patient consent for publication Not required.
Ethics approval This research was approved by the Department of Veterans' Affairs's Human Research Ethics Committee, and the University of South Australia's Human Research Ethics Committee.

Provenance and peer review Not commissioned; externally peer reviewed.

Data availability statement No data are available. The authors have full control of all primary data in conjunction with the Australian Government's Department of Veterans' Affairs (DVA). Access to data would be subsequent to permission from DVA.

Open access This is an open access article distributed in accordance with the Creative Commons Attribution Non Commercial (CC BY-NC 4.0) license, which permits others to distribute, remix, adapt, build upon this work non-commercially, and license their derivative works on different terms, provided the original work is properly cited, appropriate credit is given, any changes made indicated, and the use is non-commercial. See: http://creativecommons.org/licenses/by-nc/4.0/.

ORCID iD

Anna K Moffat http://orcid.org/0000-0001-6828-1564

\section{REFERENCES}

1 Payne RL, Martin ML. The epidemiology and management of skin tears in older adults. Ostomy Wound Manage 1990;26:26-37.

2 Rayner R, Carville K, Leslie G, et al. A review of patient and skin characteristics associated with skin tears. J Wound Care 2015;24:406-14

3 Carville K, Leslie G, Osseiran-Moisson R, et al. The effectiveness of a twice-daily skin-moisturising regimen for reducing the incidence of skin tears. Int Wound J 2014;11:446-53.

4 Finch K, Osseiran-Moisson R, Carville K, et al. Skin tear prevention in elderly patients using twice daily moisturiser. Wound Pract Res 2018;26:99-109.

5 Eccles M, Grimshaw J, Walker A, et al. Changing the behavior of healthcare professionals: the use of theory in promoting the uptake of research findings. J Clin Epidemiol 2005;58:107-12.

6 Pronovost PJ, Berenholtz SM, Needham DM. Translating evidence into practice: a model for large scale knowledge translation. BMJ 2008;337:a1714-965.

7 Cialdini RB, Goldstein NJ. Social influence: compliance and conformity. Annu Rev Psychol 2004;55:591-621.

8 Pratt NL, Kalisch Ellett LM, Sluggett JK, et al. Commitment questions targeting patients promotes uptake of under-used health services: findings from a national quality improvement program in Australia. Soc Sci Med 2015;145:1-6.

9 Roughead EE, Kalisch Ellett LM, Ramsay EN, et al. Bridging evidence-practice gaps: improving use of medicines in elderly Australian veterans. BMC Health Serv Res 2013;13:514-28.

10 Organisation WH. International statistical classification of diseases and related health problems (10th revision), Australian modification. Geneva: World Health Organisation, 2016.

11 Bandura A. Health promotion from the perspective of social cognitive theory. Psychol Health 1998;13:623-49.

12 Prochaska JO, Velicer WF, Rossi JS, et al. Stages of change and decisional balance for 12 problem behaviors. Health Psychol 1994;13:39-46.

13 Porter CM. Revisiting precede-proceed: a leading model for ecological and ethical health promotion. Health Educ J 2016;75:753-64.

14 Thaler R, Sunstein C. Nudge: improving decisions about health, wealth, and happiness. New Haven: Yale University Press, 2008.

15 Ageing AGDoHa. Schedule of pharmaceutical benefits. PBS for health professionals, 2016. Available: http://www.pbs.gov.au

16 De Hert M, Falissard B, Mauri M, et al. P.3.c.071 epidemiological study for the evaluation of metabolic disorders in patients with schizophrenia: the Meteor study. Eur Neuropsychopharmacol 2008;18:S444-5.

17 Wagner AK, Soumerai SB, Zhang F, et al. Segmented regression analysis of interrupted time series studies in medication use research. J Clin Pharm Ther 2002;27:299-309.

18 University of South Australia. Australian government department of Veterans' Affairs health claims database, 2019. 